\title{
FLOW-INVARIANT DOMAINS OF HÖLDER CONTINUITY FOR NONLINEAR SEMIGROUPS ${ }^{1}$
}

\author{
ANDREW T. PLANT
}

ABSTRACT. Let $S(t)$ be a nonlinear semigroup, on Banach space $X$, generated by an accretive set $A$. The set of $x$ in $X$ such that $t \rightarrow S(t) x$ is Hölder continuous, with Hölder exponent $\sigma \epsilon(0,1]$, is flow-invariant and is characterised by the behaviour of the map $\lambda \rightarrow(I+\lambda A)^{-1} x$ at $\lambda=0$.

0 . Introduction. Let $D$ be a subset of Banach space $X$, and $\vec{D}$ its closure. Let $S(t)$ be a strongly continuous semigroup on $\bar{D}$. That is $S:[0, \propto)$ $\times \bar{D} \rightarrow \bar{D}, S(t+s)_{x}=S(t) S(s)_{x}$ and $S(t) x \rightarrow S(0)_{x}=x$ as $t \rightarrow 0$. Suppose further

$$
\|S(t) x-S(t) y\| \leq e^{\omega t}\|x-y\| .
$$

That is $S \in Q_{\omega}(\bar{D})[4]$. For $0<\sigma \leq 1$ define

$$
D^{\sigma}=\left\{x \in \bar{D}: \limsup _{t \rightarrow 0} t^{-\sigma}\|x-S(t) x\|<\infty\right\}
$$

then clearly $D^{\sigma}$ is flow-invariant, and for each $x \in D^{\sigma}, T<\propto$, there exists $K<\infty$ such that $\|S(t) x-S(s) x\| \leq K|t-s|^{\sigma}$ for $0 \leq s, t \leq T$.

It is the purpose of this paper to characterise $D^{\sigma}$ by the behaviour of the infinitesimal generator of $S$. The result, Theorem II, is quite natural and extends some results of Crandall [3] where the generalized domain $D^{1}$ is considered.

1. Preliminaries. Let $X$ be a Banach space, and $\mathscr{G}(\omega)$ denote the set of subsets $A$ of $X \times X$ such that $A+\omega I$ is accretive. Let $D=D(A)=$ $\{x: A x \neq \varnothing\}$ and $J_{\lambda}=(I+\lambda A)^{-1}$. Accretive sets are defined in [4] where the following generation theorem is proved.

Theorem I. Let $A \in \mathbb{G}(\omega)$. Suppose there exists $\lambda_{0}>0$ such that $\lambda_{0} \omega<1$ and

$$
R(I+\lambda A) \supset \bar{D} \quad \text { for } 0<\lambda \leq \lambda_{0}
$$

then

Received by the editors August 5, 1974.

AMS (MOS) subject classifications (1970). Primary 34G05; Secondary 47H15.

Key words and phrases. Banach space, nonlinear semigroup, accretive set, flow-invariant, Hölder continuous.

${ }^{1}$ This research was supported by the British Science Research Council. 


$$
S(t) x=\lim _{n \rightarrow \infty} J_{t / n}^{n} x
$$

exists for $x \in \bar{D}$, and moreover $S \in Q_{\omega}(\bar{D})$.

Let $\left(x, x^{*}\right)$ denote the value of $x^{*} \in X^{*}$ at $x \in X$, and $F(x)=\left\{x^{*} \in X^{*}\right.$ : $\left.\left(x, x^{*}\right)=\|x\|^{2}=\left\|x^{*}\right\|^{2}\right\}$. For $x, y \in X$ define $\langle x, y\rangle_{+}=\sup \left\{\operatorname{Re}\left(x, y^{*}\right)\right.$ :

$\left.y^{*} \in F(y)\right\}$. Some properties of this function and the corresponding $\langle x, y\rangle$ _ are given in [4, Lemma 2.16] and [5, p. 74]. In particular for $x, y \in X$ and $\alpha \in \mathbf{R}$

$$
\left|\langle x, y\rangle_{+}\right| \leq\|x\|\|y\|, \quad\langle x+\alpha y, y\rangle_{+}=\langle x, y\rangle_{+}+\alpha\|y\|^{2} .
$$

Our proof of Theorem II in the next section is based on the following result.

Lemma 1.1. Let $A$ and $S(t)$ be as in Theorem I. Let $x \in \bar{D}$ and $\left[x_{0}, y_{0}\right] \in A$ then

$$
\left\|x_{0}-S(t) x\right\|^{2}-\left\|x_{0}-x\right\|^{2} \leq 2 \int_{0}^{t}\left\langle y_{0}+\omega\left(x_{0}-S(\tau) x\right), x_{0}-S(\tau) x\right\rangle_{+} d \tau .
$$

The proof of (1.4) for the case $\omega=0$ is given by [7, Equation (2.10)]. The case for general $\omega$ is easy to deduce from [5, Equation (3.8)]. Replacing $x$ by $S(s) x$ gives

$$
H(t)=\int_{0}^{t}\left\langle y_{0}+\omega\left(x_{0}-S(\tau) x\right), x_{0}-S(\tau) x\right\rangle_{+} d \tau-1 / 2\left\|x_{0}-S(t) x\right\|^{2}
$$

is nondecreasing.

2. Hölder continuous domains. We characterise $D^{\sigma}$ by defining the following two functions on $\bar{D}$.

$$
|S x|_{\sigma}=\limsup _{t \rightarrow 0} t^{-\sigma}\|x-S(t) x\|, \quad|A x|_{\sigma}=\limsup _{\lambda \rightarrow 0} \lambda^{-\sigma}\left\|x-J_{\lambda} x\right\| .
$$

Theorem II. Suppose $A \in \mathbb{G}(\omega)$ and (1.1) holds. Let $S(t)$ be defined by (1.2). Then for $0<\sigma \leq 1$,

$$
\begin{gathered}
(1 / 3)|S x|_{\sigma} \leq|A x|_{\sigma} \leq 3|S x|_{\sigma}, \\
D^{\sigma}=\left\{x \in \bar{D}:|S x|_{\sigma}<\infty\right\}=\left\{x \in \bar{D}:|A x|_{\sigma}<\infty\right\} .
\end{gathered}
$$

Proof. Clearly (2.2) is a consequence of (2.1). To avoid considering separate cases we may assume in full generality that $\omega>0$. Let $\lambda>0$ and $\lambda \omega<1$, and in (1.5) set $x_{0}=J_{\lambda} x, y_{0}=\lambda^{-1}\left(x-x_{0}\right)$. If $z(\tau)=\left\|x_{0}-s(\tau) x\right\|$ and $f(\tau)=\lambda^{-1}[\|x-S(\tau) x\|-(1-\lambda \omega) z(\tau)]$ then by $(1.3)$

$$
\begin{aligned}
\lambda\left\langle y_{0}+\omega\right. & \left.\left(x_{0}-S(\tau) x\right), x_{0}-S(\tau) x\right\rangle_{+} \\
& =\left\langle x-S(\tau) x-(1-\lambda \omega)\left(x_{0}-S(\tau) x\right), x_{0}-S(\tau) x\right\rangle_{+} \\
& =\left\langle x-S(\tau) x, x_{0}-S(\tau) x\right\rangle_{+}-(1-\lambda \omega) z(\tau)^{2} \leq \lambda f(\tau) z(\tau)
\end{aligned}
$$


Therefore $\int_{0}^{t} f(\tau) z(\tau) d \tau-1 / 2 z(t)^{2}$ is nondecreasing. Consequently, if $z(t) \neq 0,0 \leq f(t)-D z(t)$, where $D$ represents any of the four Dini derivates. However, if $D^{-} z(t)$ (resp. $D_{-} z(t)$ ) represents the upper (resp. lower) left-hand derivate of $z$ at $t>0$, then $z(t)=0$ implies $D_{-} z(t) \leq D^{-} z(t) \leq 0 \leq f(t)$. Consequently for all $t>0,0 \leq f(t)-D_{-} z(t)=D^{-}\left[\int_{0}^{t} f(\tau) d \tau-z(t)\right]$; and so by a classical result, e.g. [8, p. 84], the bracketed term is nondecreasing. In particular $z(t)-z(0) \leq \int_{0}^{t} f(\tau) d \tau$.

Now set $x(t)=\|x-S(t) x\|, y(\lambda)=\left\|x-J_{\lambda} x\right\|=\left\|x-x_{0}\right\|=z(0)$. Then $|x(t)-y(\lambda)| \leq z(t)$ and consequently

$$
|x(t)-y(\lambda)|-y(\lambda) \leq \lambda^{-1} \int_{0}^{t} x(\tau)-(1-\lambda \omega)|x(\tau)-y(\lambda)| d \tau .
$$

The modulus signs may be removed to give four inequalities of which one is trivial. We use two of the remaining three. First

$$
x(t)-2 y(\lambda) \leq \lambda^{-1} \int_{0}^{t} \lambda \omega x(\tau)+(1-\lambda \omega) y(\lambda) d \tau
$$

which, after integrating the constant term and applying Gronwall's lemma [6, p. 283], gives

$$
x(t) \leq[2+(1+\lambda \omega)(\exp (\omega t)-1) / \lambda \omega] y(\lambda) .
$$

Now set $\lambda=t$, divide by $t^{\sigma}$ and let $t \rightarrow 0$ to obtain the first inequality in (2.1). Returning to (2.3),

$$
-x(t) \leq \lambda^{-1} \int_{0}^{t}(2-\lambda \omega) x(\tau)-(1-\lambda \omega) y(\lambda) d \tau
$$

which rearranges to

$$
(1-\lambda \omega) y(\lambda) \leq(\lambda / t) x(t)+(2-\lambda \omega) t^{-1} \int_{0}^{t} x(\tau) d \tau .
$$

For the second inequality in (2.1) we may assume $|S x|_{\sigma}<\infty$, so $L(t)=$

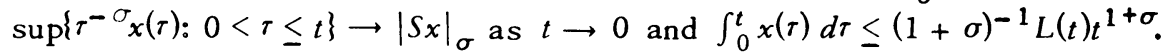
Then setting $\lambda=t$ in (2.5) gives

$$
(1-t \omega) y(t) \leq x(t)+(2-t \omega)(1+\sigma)^{-1} L(t) t^{\sigma}
$$

and the required inequality follows. This completes the proof.

From (2.4) and (2.5) we easily deduce global estimates:

Corollary. If $\rho(t)=\left(e^{t}-1\right) / t(t>0), \rho(t)=1(t \leq 0), \omega^{+}=\max \{\omega, 0\}$ and $\lambda \omega<1$ then

$$
\|x-S(t) x\| \leq\left[2+\left(1+\lambda \omega^{+}\right) \rho(\omega t) t / \lambda\right]\left\|x-J_{\lambda} x\right\|,
$$

$$
\left(1-\lambda \omega^{+}\right)\left\|x-J_{\lambda} x\right\| \leq(\lambda / t)\|x-S(t) x\|+\left(2-\lambda \omega^{+}\right) t^{-1} \int_{0}^{t}\|x-S(\tau) x\| d \tau .
$$

In particular if $\omega=0$

$$
\frac{1}{3}\|x-S(t) x\| \leq\left\|x-J_{t} x\right\| \leq\|x-S(t) x\|+\frac{2}{t} \int_{0}^{t}\|x-S(\tau) x\| d \tau .
$$


Remark 1. The first inequality in (2.8) was announced by D. Brézis [2] for the case $X$ is a Hilbert space. The general case has the following simple proof, for which we thank the referee:

$$
\begin{aligned}
\|x-S(t) x\| & \leq\left\|x-J_{t} x\right\|+\left\|J_{t} x-S(t) J_{t} x\right\|+\left\|S(t) J_{t} x-S(t) x\right\| \\
& \leq 2\left\|x-J_{t} x\right\|+\left\|J_{t} x-S(t) J_{t} x\right\|,
\end{aligned}
$$

but

$$
\left\|J_{t} x-S(t) J_{t} x\right\| \leq t\left\|A_{t} x\right\|=\left\|x-J_{t} x\right\| .
$$

The second inequality in (2.8) should be compared with $\left\|x-J_{t} x\right\| \leq$ $3\|x-S(t) x\|$ obtained by Brézis for the case $X$ is a Hilbert space and $A$ is a gradient. In the general case periodic orbits are possible, and the Brézis estimate fails. For example consider the rotation group in $\mathbf{R}^{2}$.

Finally, if $X$ is a Hilbert space, one deduces from Theorem 4 of [2] with $p=\propto$ that

$$
\frac{1}{3} \leq\left(\sup _{0<t \leq 1} t^{-\sigma}\left\|x-J_{t} x\right\|\right) /\left(\sup _{0<t \leq 1} t^{-\sigma}\|x-S(t) x\|\right) \leq 6 .
$$

(2.8) gives the same result with 6 replaced by $(3+\sigma) /(1+\sigma) \leq 3$.

Remark 2. Our method can easily be used to improve estimates (2.1). In (2.4), (2.5) set $\lambda=\alpha t$, take the limit as before and then minimize with respect to $\alpha>0$. This gives

$$
\gamma(\sigma)|S x|_{\sigma} \leq|A x|_{\sigma} \leq\left[(1+\sigma)^{1-\sigma} \gamma(\sigma)\right]^{-1}|S x|_{\sigma}
$$

where $\gamma(\sigma)=\sigma(1 / 2(1-\sigma))^{1-\sigma}(\sigma<1), \gamma(1)=1$, which is best possible at $\sigma=$ 1 (i.e. $|S x|_{1}=|A x|_{1}$ ) (proved by Crandall [3]). In the next section we give an example where $|A x|_{1 / 2}<|S x|_{1,2}$.

Remark 3. The results in this section have wider applicability than we have so far indicated. Condition (1.5) expresses the fact that $S(t) x$ is, in the terminology of $\mathrm{Ph}$. Bénilan [1], a solution intégrale of $(d u / d t)+A u \ni 0$. Consequently our results apply to such solutions, provided $x \in R(I+\lambda A)$.

3. An example. We consider an example of Webb [9]. Let $X$ be the Banach space of bounded uniformly continuous real-valued functions on $[0, \infty)$ with supremum norm. Let $A f=-f^{\prime}, D=D(A)=\left\{f: f^{\prime} \in X\right\}$. Then $A$ is closed, linear, densely defined and $m$-accretive. The corresponding semigroup is translations, $(S(t) f)(s)=f(s+t)$, so $D^{\sigma}$ is the subspace of uniformly Hölder continuous functions with Hölder exponent $\sigma_{\bullet}$. Moreover

$$
\left(J_{\lambda} f\right)(t)=\lambda^{-1} \int_{t}^{\infty} f(s) \exp [(t-s) / \lambda] d s .
$$

Now set $f(t)=(1-t)^{1 / 2}(t<1), f(t)=0(t \geq 1)$. So $|S f|_{1 / 2}=1$, and it is an easy computation to show 


$$
\begin{aligned}
\lambda^{-1 / 2}\left(f(t)-\left(J_{\lambda} f\right)(t)\right) & =\int_{0}^{y} \exp \left(x^{2}-y^{2}\right) d x \quad(t<1) \\
& =0 \quad(t \geq 1)
\end{aligned}
$$

where $y=(1-t)^{1 / 2} \lambda^{-1 / 2}$. Therefore

$$
\lambda^{-1 / 2}\left\|f-J_{\lambda} f\right\|=\sup _{y} \int_{0}^{y} \exp \left(x^{2}-y^{2}\right) d x
$$

where sup is taken over the range $0 \leq y \leq \lambda^{-1 / 2}$. Thus

But

$$
|A f|_{1 / 2}=\sup _{0 \leq y} \int_{0}^{y} \exp \left(x^{2}-y^{2}\right) d x .
$$

$$
\int_{0}^{y} \exp \left(x^{2}-y^{2}\right) d x \leq e^{-y^{2}} \int_{0}^{y} e^{x y} d x=\left(1-e^{-y^{2}}\right) / y<(2 / e)^{1 / 2} .
$$

Therefore

$$
|A f|_{1 / 2} \leq(2 / e)^{1 / 2}<1=|S f|_{1 / 2} \text {. }
$$

Acknowledgments. I thank Professor J. A. Goldstein for first drawing my attention to the work on D. Brézis [2], and the referee for explicitly showing how my results relate to those of Brézis.

\section{REFERENCES}

1. Ph. Bénilan, Solutions intégrales d'equations d'évolution dans un espace de Banach, C. R. Acad. Sci. Paris Sér. A-B 274 (1972), A47-A50. MR 45 \#9212.

2. D. Brézis, Classes d'interpolation associées à un opérateur monotone, C. R. Acad. Sci. Paris Sér. A-B 276 (1973), A1553-A 1556.

3. M. G. Crandall, A generalized domain for semigroup generators, M.R.C. Technical Report \#1189, University of Wisconsin, Madison, Wis.

4. M. G. Crandall and T. M. Liggett, Generation of semi-groups of nonlinear transformations on general Banach spaces, Amer. J. Math. 93 (1971), 265-298. MR 44 \#4563.

5. M. G. Crandall and A. Pazy, Nonlinear evolution equations in Banach spaces, Israel J. Math. 11 (1972), 57-94. MR 45 \#9214.

6. J. Dieudonné, Fondements de l'analyse moderne, Pure and Appl. Math., vol. 10, Academic Press, New York, 1960. MR $22 \# 11074$.

7. I. Miyadera, Some remarks on semi-groups of nonlinear operators, Tôhoku Math. J. (2) 23 (1971), 245-258. MR 45 \#5805.

8. H. L. Royden, Real analysis, Macmillan, New York 1963. MR $27 \# 1540$.

9. G. F. Webb, Continuous nonlinear pertubations of linear accretive operators in Banach spaces, J. Functional Analysis 10 (1972), 191-203.

FLUID MECHANICS RESEARCH INSTITUTE, UNIVERSITY OF ESSEX, COLCHESTER CO4 3SQ, ENGLAND 